\title{
ULTRAVIOLET AND EXTREME-ULTRAVIOLET EMISSIONS AT THE FLARE FOOTPOINTS OBSERVED BY ATMOSPHERE IMAGING ASSEMBLY
}

\author{
Jiong Qiu ${ }^{1}$, Zoe Sturrock ${ }^{2}$, Dana W. Longcope ${ }^{1}$, James A. KlimchuK $^{3}$, And Wen-JuAn Liu ${ }^{1}$ \\ ${ }^{1}$ Department of Physics, Montana State University, Bozeman, MT 59717-3840, USA \\ ${ }^{2}$ Department of Applied Mathematics, University of St. Andrews, UK \\ ${ }^{3}$ NASA Goddard Space Flight Center, Greenbelt, MD 20771, USA \\ Received 2013 April 1; accepted 2013 June 21; published 2013 August 9
}

\begin{abstract}
A solar flare is composed of impulsive energy release events by magnetic reconnection, which forms and heats flare loops. Recent studies have revealed a two-phase evolution pattern of UV $1600 \AA$ emission at the feet of these loops: a rapid pulse lasting for a few seconds to a few minutes, followed by a gradual decay on timescales of a few tens of minutes. Multiple band EUV observations by the Atmosphere Imaging Assembly further reveal very similar signatures. These two phases represent different but related signatures of an impulsive energy release in the corona. The rapid pulse is an immediate response of the lower atmosphere to an intense thermal conduction flux resulting from the sudden heating of the corona to high temperatures (we rule out energetic particles due to a lack of significant hard X-ray emission). The gradual phase is associated with the cooling of hot plasma that has been evaporated into the corona. The observed footpoint emission is again powered by thermal conduction (and enthalpy), but now during a period when approximate steady-state conditions are established in the loop. UV and EUV light curves of individual pixels may therefore be separated into contributions from two distinct physical mechanisms to shed light on the nature of energy transport in a flare. We demonstrate this technique using coordinated, spatially resolved observations of UV and EUV emissions from the footpoints of a C3.2 thermal flare.
\end{abstract}

Key words: magnetic reconnection - Sun: flares - Sun: UV radiation

Online-only material: color figures

\section{INTRODUCTION}

The source of flare energy is magnetic, but the ultimate form of the flare energy output is radiation of all kinds: lines and continua by thermal and non-thermal particles. The bulk of $\mathrm{X}$-ray and EUV radiation is produced in flare coronal loops, whereas enhanced optical, UV, and hard X-ray emissions are usually observed at the footpoints of these flare loops. Flare plasmas and particles are magnetically confined to be able to communicate mainly along the loop from its lower-atmosphere root to the corona. Therefore, various radiation signatures along a flare loop are coherently coupled by physics governing energetics and dynamics of magnetized flare plasmas.

In general, we may separate the energy release process along a flare loop into the heating phase and the cooling phase. A series of hydrodynamic responses takes place in an impulsively heated flaring atmosphere. A downward heat flux or energetic particle beam generates a localized pressure pulse that drives bi-directional flow: an evaporation upflow into the corona and a condensation downflow into the chromosphere (Canfield 1986). Evaporation sends heated plasma into the corona, which then cools as it radiates in X-ray and EUV wavelengths. Note that thermal conduction cooling of the evaporated material drives further evaporation, but this is a quasi-steady process, different from the initial explosive evaporation. Finally, the late stage of cooling is dominated by radiation and involves a slow draining of the material back onto the chromosphere. This process of flare loop evolution is often demonstrated by observations showing that bulk X-ray and EUV emissions in the corona are delayed with respect to the impulsively rising hard X-ray, UV, and optical emissions from the lower atmosphere.

Numerous spectroscopic observations have unraveled dynamics in the early (heating) phase of the flare, showing upflows of up to a few hundred kilometers per second in hot lines formed at a few million degrees (Antonucci et al. 1982; Doschek et al. 1992; Culhane et al. 1992; Bentley et al. 1994), as well as downflows of several tens of kilometers per second in the chromospheric $\mathrm{H} \alpha$ line (Ichimoto \& Kurokawa 1984; Canfield \& Metcalf 1987; Schmieder et al. 1987; Canfield et al. 1987, 1990a, 1990b; Zarro et al. 1988; Wuelser \& Marti 1989; Wuelser et al. 1994; Ding et al. 1995). Spectroscopic observations by recent missions such as the Coronal Diagnostic Spectrometer (CDS; Harrison et al. 1995) and the EUV Imaging Spectrometer (EIS; Culhane et al. 2007) have also identified these dynamical phenomena in UV and EUV lines at the feet of flare loops, where sometimes hard X-ray sources are located (Milligan et al. 2006a, 2006b; Milligan \& Dennis 2009; Watanabe et al. 2010; Del Zanna et al. 2011; Graham et al. 2011). It takes a rather short time for energy flux carried by either non-thermal particles or thermal conduction to reach the lower atmosphere and enhance UV and optical emissions (Fisher et al. 1985; Canfield \& Gayley 1987). Therefore, the impulsive and dynamic behavior of radiation at the lower atmosphere, usually ahead of significant coronal emissions, is registered as prompt signatures of flare energy release.

On the other hand, during the cooling phase, observations of some stellar flares have shown that emissions in a few optical and UV bands appear to decay rather gradually at a rate very similar to the timescale of coronal radiation (Hawley \& Fisher 1992; Hawley et al. 2003). Similar behavior of UV light curves was observed in some flares by the Solar Maximum Mission (e.g., Cheng \& Pallavicini 1987). With high-resolution observations by the Transition Region And Corona Explorer (TRACE; Handy et al. 1999) and the Atmosphere Imaging Assembly (AIA; Lemen et al. 2012), Qiu et al. (2010, 2012), Cheng et al. (2012), and Liu et al. (2013) have also found that the broadband $1600 \AA$ 
UV emission from individual pixels $\left(1^{\prime \prime} \times 1^{\prime \prime}\right)$ exhibits two-phase evolution characterized by a rapid rise and a gradual decay. During the cooling phase, conductive flux continuously flows from the corona toward the lower atmosphere-the transition region and chromosphere-which cools off by radiation. It is therefore considered that the prolonged decay in the lower atmosphere emission is coupled with coronal evolution, and may serve as a coronal "pressure gauge" (Fisher 1987; Griffiths et al. 1998; Hawley et al. 2003).

Separating the radiative signatures from the footpoint of $a$ single loop into two distinct physical contributions provides crucial observational constraints to flare models. Fisher \& Hawley (1990) modeled an observed solar flare with a heating rate assumed to have the same time profile of the observed hard $\mathrm{X}$-ray light curve. Quite a few solar flare studies followed a similar approach using (spatially unresolved) hard X-ray light curves or energy flux converted from spectral analysis to infer impulsive energy release rate in the flare loop (e.g., Raftery et al. 2009). Taking advantage of high-resolution UV imaging observations, Qiu et al. (2012) and Liu et al. (2013) recently modeled heating of thousands of flare loops (with nominal cross section of $1^{\prime \prime} \times 1^{\prime \prime}$ ) using heating rates inferred from the rise phase of the UV emission at the feet of these flare loops. Using UV signatures to build heating rates, these studies not only resolve heating in individual loops but are not confined to flares that have significant thick-target hard X-ray emissions. It should be noted that the subsequent decay of the UV emission at these same feet, which is considered to be governed by evolution of the overlying flaring corona, should depend on the heating history. Along this line, Liu et al. (2013) conducted modeling and analysis of an M8.0 flare and computed UV emission in the cooling phase. The result has shown, for the first time, that the computed UV emission is in good agreement with the observed UV flux and both decay at the same rate.

In this paper, we report UV and EUV observations of a C3.2 flare observed by AIA on 2010 August 1. It is found that the flare EUV emission at the footpoints exhibits a two-phase evolution similar to the UV emission. We speculate that these EUV emissions are also generated in the lower atmosphere such as the transition region, which is impulsively heated and then cools down on coronal evolution timescales. This same notion was addressed in a few previous studies. While modeling active region loops, Patsourakos \& Klimchuk (2008) and Klimchuk $(2009,2012)$ have shown that the transition region emission at the base of coronal loops contributes significantly to the total emission budget in EUV $171 \AA$ such as observed by TRACE. Recently, Brosius \& Holman (2012) suggested that the simultaneous EUV emissions observed by AIA during the early phase of a B4.8 flare were produced by loweratmosphere plasmas of a few hundred thousand degrees. On the other hand, some recent observations by the Soft X-ray Telescope (SXT; Tsuneta et al. 1991; e.g., Mrozek \& Tomczak 2004) and EIS (e.g., Milligan 2011; Graham et al. 2013) have revealed high-temperature emissions of up to $8 \mathrm{MK}$ at the flare footpoints during the impulsive phase. In those events, hard X-ray emissions were also found at the footpoints, and chromospheric evaporation is considered to be driven by precipitating non-thermal particles. In this study, we will discuss the origin of the footpoint EUV emissions in this C3.2 flare and their implication on flare modeling. The following section gives an overview of the flare, followed by observations of the spatially resolved flare footpoint emissions in UV and EUV bands. In Section 4, we estimate UV and EUV emissions during the decay phase using a simple conductive heating model, and conclusions and discussions are presented in the last section.

\section{OBSERVATIONS}

In this paper, we exclusively study the rise and decay of UV and EUV emissions in flare footpoints identified from AIA observations of a C3.2 flare on 2010 August 1. A preliminary analysis of emissions from the coronal loops of the same flare observed by AIA and GOES is presented in Qiu et al. (2012). Figure 1 shows the light curves of the total data counts summed in the active region in a few UV and EUV bands observed by AIA. For clarity of presentation, in the plot, the minimum value is subtracted from each light curve, which is then normalized to its maximum. We note that whereas emissions in UV, soft X-ray, and EUV $94 \AA$ band rise during the flare, the EUV $171 \AA$ emission first decreases during the rise phase of the flare and then increases $2 \mathrm{hr}$ later. The early attenuation of the EUV $171 \AA$ emission is caused by disruption and disappearance of active region loops at the onset of the flare, which contributes to coronal dimming typically observed in this wavelength (Harra et al. 2007; Qiu et al. 2007; also see Hock et al. 2013, for recent observations).

As shown in Figure 1, the flare is a long-duration event, with coronal radiation in soft X-ray and then subsequently EUV temperatures lasting for nearly four hours. Enhanced emissions at UV $1600 \AA$ band last for two hours. Throughout the flare, UV or EUV flux observed by AIA is not saturated in any band, and the exposure time at any single band was a constant; therefore, the flare is a good candidate for quantitative analysis. RHESSI observations of this flare show gradual X-ray emission up to $20 \mathrm{keV}$ similar to the GOES light curve, suggesting that the flare probably does not have significant non-thermal emissions.

Flare emission in the UV $1600 \AA$ broadband is dominated by $\mathrm{C}$ IV line emission, which is an optically thin line formed at $10^{5} \mathrm{~K}$, the temperature of the upper chromosphere and transition region. Enhancement of this emission is observed at the feet of the flare loops, thereby forming the classic flare ribbons. AIA also observes at the UV $1700 \AA$ broadband, which mostly reflects the flare-enhanced UV continuum emission at the flare footpoints. Past spectral observations suggest that UV continuum in these wavelengths is formed at the temperature minimum and thus characterized by temperatures of 4400-4700 K in quiescent or active regions (Brekke \& Kjeldseth-Moe 1994). Continuum enhancement during a flare is characterized by brightness temperatures up to $5400 \mathrm{~K}$ (Cook \& Brueckner 1979). Cook $\&$ Brueckner (1979) also reported that the decay time of this increased brightness temperature is comparable to the soft X-ray decay time. Given the large column depth of the temperature minimum region, these enhancements are not readily explained by direct heating from either thermal or non-thermal electron flux. Instead it is typically attributed to photoionization from short-wavelength emissions from above (Machado \& Henoux 1982; Phillips et al. 1992; Doyle \& Phillips 1992). This close causal link between the enhancements of C IV and UV continuum explains the nearly identical morphology observed in the $1600 \AA$ and $1700 \AA$ images.

Assuming the continuum enhancement to be the same in both the $1700 \AA$ and $1600 \AA$ bands, although characterized by different regions of the blackbody curve, we can use the former to remove the continuum from the latter. To do this, we assume that the $1700 \AA$ band is dominated by the continuum emission (Brekke et al. 1996) to estimate the brightness temperature of the enhancement in a given pixel. We then subtract an amount 


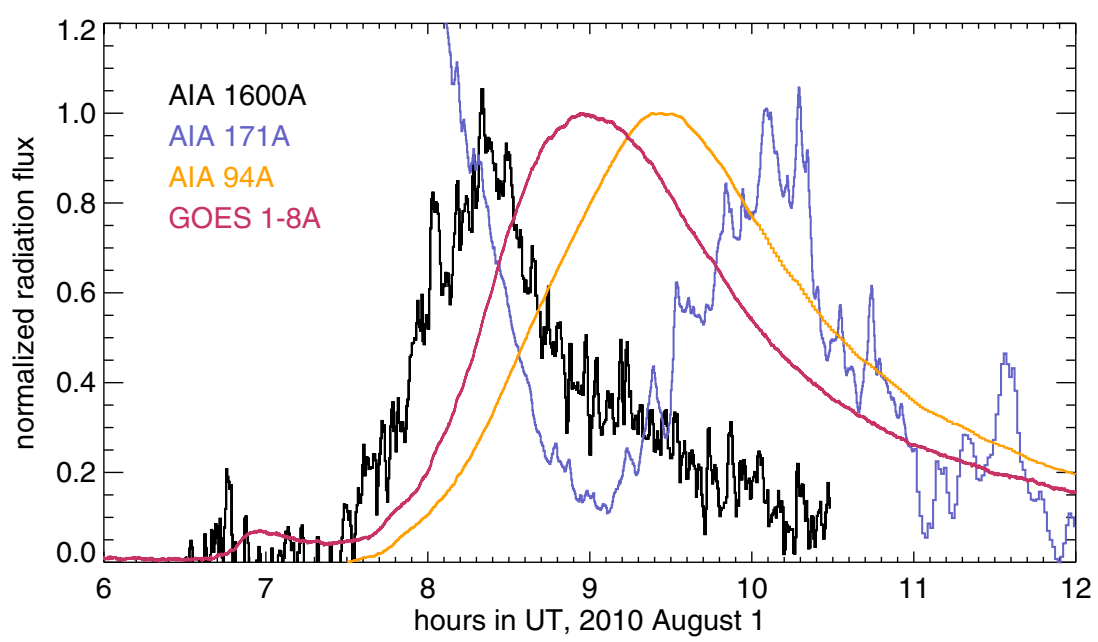

Figure 1. Light curves of the 2010 August 1 C 3.2 flare in UV $1600 \AA$ and EUV 171 and $94 \AA$ by SDO/AIA, and soft X-ray $1-8 \AA$ by GOES.

(A color version of this figure is available in the online journal.)

from the $1600 \AA$ band corresponding to the same brightness temperature. The remainder, we contend, is an estimate of the $\mathrm{C}$ IV emission from that pixel.

EUV emissions are usually produced in flare loops heated to a few tens of $\mathrm{MK}$ and then cooled to 2-3 million kelvin or even below (e.g., Reale et al. 2012). This general statement is supported by Figure 1, showing X-ray and EUV emission characteristics of different temperatures peaking at different times. Figure 2 shows the time sequence of the flare evolution observed in UV $1600 \AA$ broadband, as well as in five EUV bands at $304,193,335,94$, and $131 \AA$, which are roughly representative of increasing temperatures of coronal plasmas. The figure shows the flare to consist of brightenings in two different loop systems. A set of short loops in the north brightens first, followed by a set of long loops in the south. For the same loop(s), emissions at relatively high temperatures (in the 131 and $94 \AA$ bands, for example) occur earlier than emissions at relatively low temperatures (in the 193 and $304 \AA$ bands, for example).

Apart from EUV emissions in flare loops, these images also show impulsive rise of EUV emission coincident with the UV emission at the same location during the early phase of the flare (left column of Figure 2). These emissions arise where the flare loops, visible in EUV images minutes later, are rooted. The origin of these emissions is the focus of this study.

Images from the AIA multiple bands are rebinned to the scale of $1^{\prime \prime} .2 \times 1$. 2 and are co-aligned with each other with subarcsecond accuracy. Spatially resolved light curves, in units of data number (DN) per second per pixel, are obtained in these bands. In the following analysis, we select the brightest UV footpoint pixels observed in $1600 \AA$ that exhibit strong emission, or more specifically those pixels with a count rate greater than five times the median count rate $\left(I_{q}=71 \mathrm{DN} \mathrm{s}^{-1}\right)$ of the quiescent region for more than 3 minutes. These pixels account for $50 \%$ of all flaring pixels analyzed in Qiu et al. (2012), but since these are the brighter half, their total emission predominates the total UV emission of the flare.

\section{UV AND EUV EMISSIONS AT THE FOOTPOINTS OF FLARING LOOPS}

\subsection{UV Emissions}

The top panels of Figure 3 show an example of the UV 1600 (dark dashed line in both panels) and 1700 (dark solid line in the right panel) light curves from one of the brightest UV pixels. (This pixel is the brightest pixel within the small red box in the left column of Figure 2.) Most of the bright pixels exhibit a rapid rise for 5-10 minutes, followed by a gradual decay over a few tens of minutes. Such two-phase evolution was reported in stellar flares observed in a few UV lines including the C IV line (Hawley et al. 2003). Recently, Qiu et al. (2010, 2012), Cheng et al. (2012), and Liu et al. (2013) also reported such an evolution pattern in UV 1600 emissions from spatially resolved flare kernels observed by TRACE or AIA. So the two-phase evolution appears to be common in UV emissions from flare footpoints.

We also note that the observed $1700 \AA$ emission exhibits a light curve very similar to that of $1600 \AA$ : an impulsive rise and a gradual decay on timescales identical to those observed in $1600 \AA$ band. While the morphology of the two light curves is identical, they are quantitatively quite different. The $1600 \AA$ emission is enhanced by an order of magnitude over the preflare emission, while the peak $1700 \AA$ emission is only about twice the pre-flare emission. We attribute this difference to the contribution of $\mathrm{C}$ IV to the former and not the latter.

The top panels of Figure 4 show the UV light curves computed from the summed counts from all footpoint pixels identified in the UV $1600 \AA$ band. With all flaring pixels summed up, emissions in the $1600 \AA$ and $1700 \AA$ bands rise above pre-flare levels by $150 \%$ and $40 \%$, respectively.

The broadband $1600 \AA$ emission obtained by AIA includes contribution by the optically thin C IV line emission, which forms at the temperature of $10^{5} \mathrm{~K}$, the typical transition region temperature, and the UV continuum forming around $4500 \mathrm{~K}$ in the temperature minimum region. Both the line emission and the continuum emission are enhanced during the flare when the lower atmosphere is heated. AIA also takes broadband images at UV $1700 \AA$ with a few lines, whose net contribution, however, may not dominate the emission in this broadband (Brekke et al. 1996). Comparison of images obtained in these two bands therefore helps to distinguish C IV emission from the UV continuum.

To the first order, we assume that the UV continuum in both bands is formed at the same brightness temperature $T_{B}$ described by Planck's function, and that the $1700 \AA$ emission is predominantly continuum emission. Taking into account the AIA instrument response function, the $1700 \AA$ emission can 


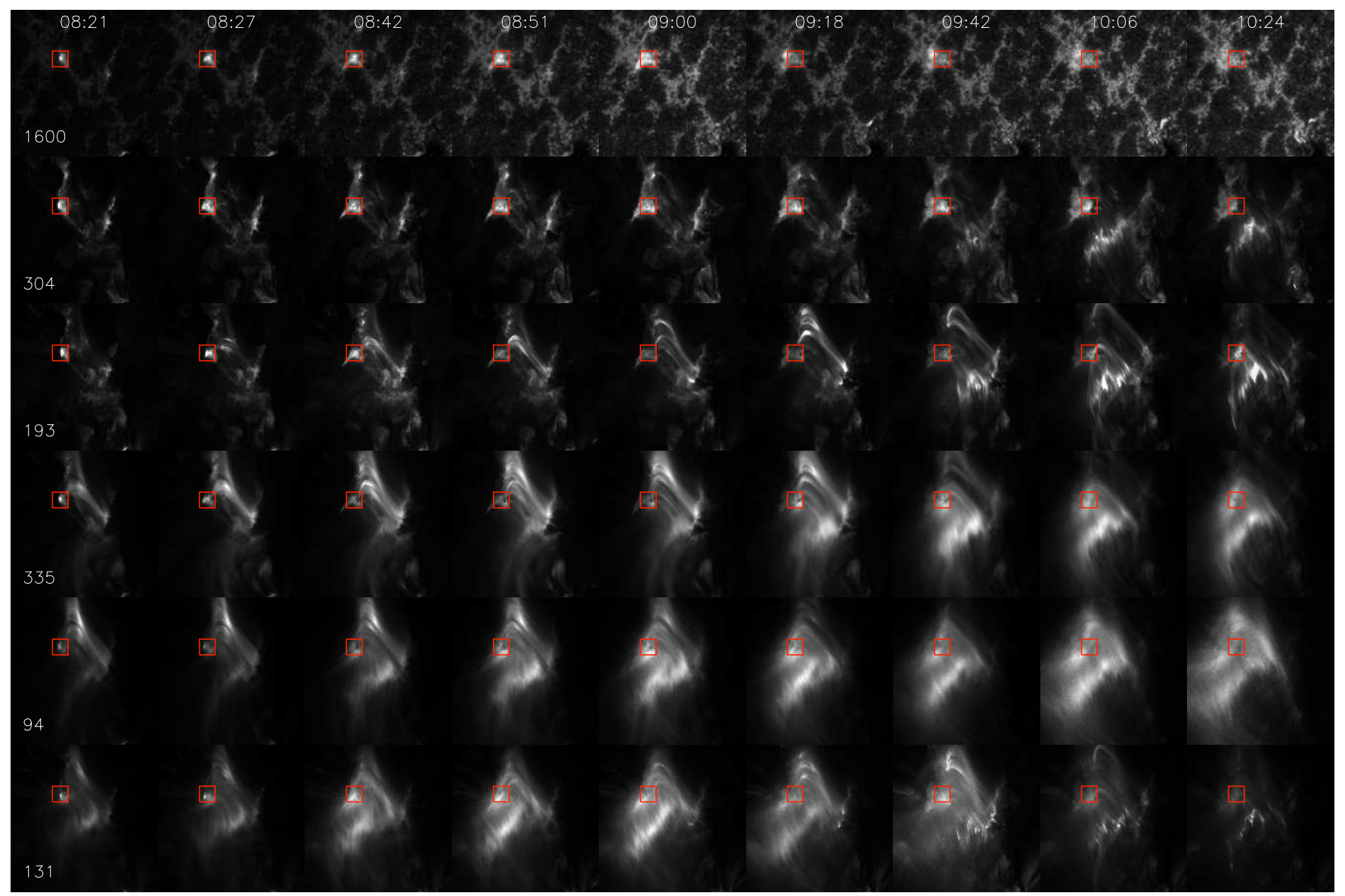

Figure 2. Evolution of the flare as observed in AIA UV $1600 \AA$ A band and EUV 94, 131, 193, 304, and 335 Åbands. Images at EUV 171 and $211 \AA \AA$ are not shown, since the flare morphology in these two bands is similar to that observed in $193 \AA$ band. The red box in the figure shows the location of the sample footpoint pixel, which is impulsively brightened and identified in the UV $1600 \AA$ images. Images of the left column show UV and EUV images at the time when this pixel is brightest; it is seen that this same pixel is brightest at all bands. Images in other columns show time evolution after the impulsive brightening at this pixel. Whereas UV $1600 \AA$ images only exhibit emission at the footpoint, all EUV images show flare loops connected at or overlapping upon this sample footpoint pixel. The times of these images are also indicated by dotted lines in Figure 3.

(A color version of this figure is available in the online journal.)

then be used to compute the brightness temperature $T_{B}$. The red curve in the top right panel of Figure 3 is the computed $T_{B}$ at the sample pixel during the flare. This temperature varies from $4800 \mathrm{~K}$ before the flare to $5200 \mathrm{~K}$ at the peak of the flare, namely the brightness temperature is raised by $400 \mathrm{~K}$ for this bright flaring pixel. These numbers are within the reasonable range in agreement with past UV spectroscopic observations of flares (Cook \& Brueckner 1979).

We then compute the continuum contribution to the $1600 \AA$ band using the same $T_{B}$ and the response function of the AIA UV filter. The calculated $1600 \AA$ continuum light curve for that same pixel is shown as the blue curve in the top left panel in the figure, together with the observed total count rate in this band, both in absolute scales. The comparison suggests that whereas the pre-flare emission in this broadband is dominated by the continuum, during the flare, the continuum emission contributes only a fraction of the total UV emission. The remainder UV emission during the flare is most likely the contribution of the C IV line (dark solid curve). In this bright pixel, the peak C IV emission is about four times the continuum emission. When summed over all flaring pixels (top left panel in Figure 4), the total CIV emission (dark solid curve) is about 1.5 times the continuum emission (blue solid curve).

We caution that the above exercise gives an estimate of the possible contributions by the continuum and the $\mathrm{C}$ IV line emissions to the UV $1600 \AA$ broadband. In this estimate, we have ignored contributions by all other lines in both the $1600 \AA$ and $1700 \AA$ ands. On the other hand, by subtracting the 1700 emission off the 1600 band, contributions of these lines are partly canceled. The estimate therefore only provides a firstorder evaluation of C IV emission in the flaring atmosphere.

\subsection{EUV Emissions}

The other panels in Figure 3 show light curves (dark solid line) in six EUV bands for comparison to the UV $1600 \AA$ A light curve (dark dashed line) from the same footpoint pixel. It is evident that the EUV emission at one pixel typically exhibits at least two peaks, and that the first peak in each EUV band is coincident with the UV emission peak. Just like the UV light curve, the first EUV peak also exhibits a two-phase evolution, a rapid rise followed by a more gradual decay, and the rise and decay timescales are entirely comparable with those of the UV light curves. The EUV filters of AIA are sensitive to plasmas with a range of temperatures including, in every case, a few hundred thousand degrees (Lemen et al. 2012). It is therefore very likely that the first EUV peak is produced the same way UV emission is produced: impulsive energy deposition from thermal conduction in the lower atmosphere followed by a 

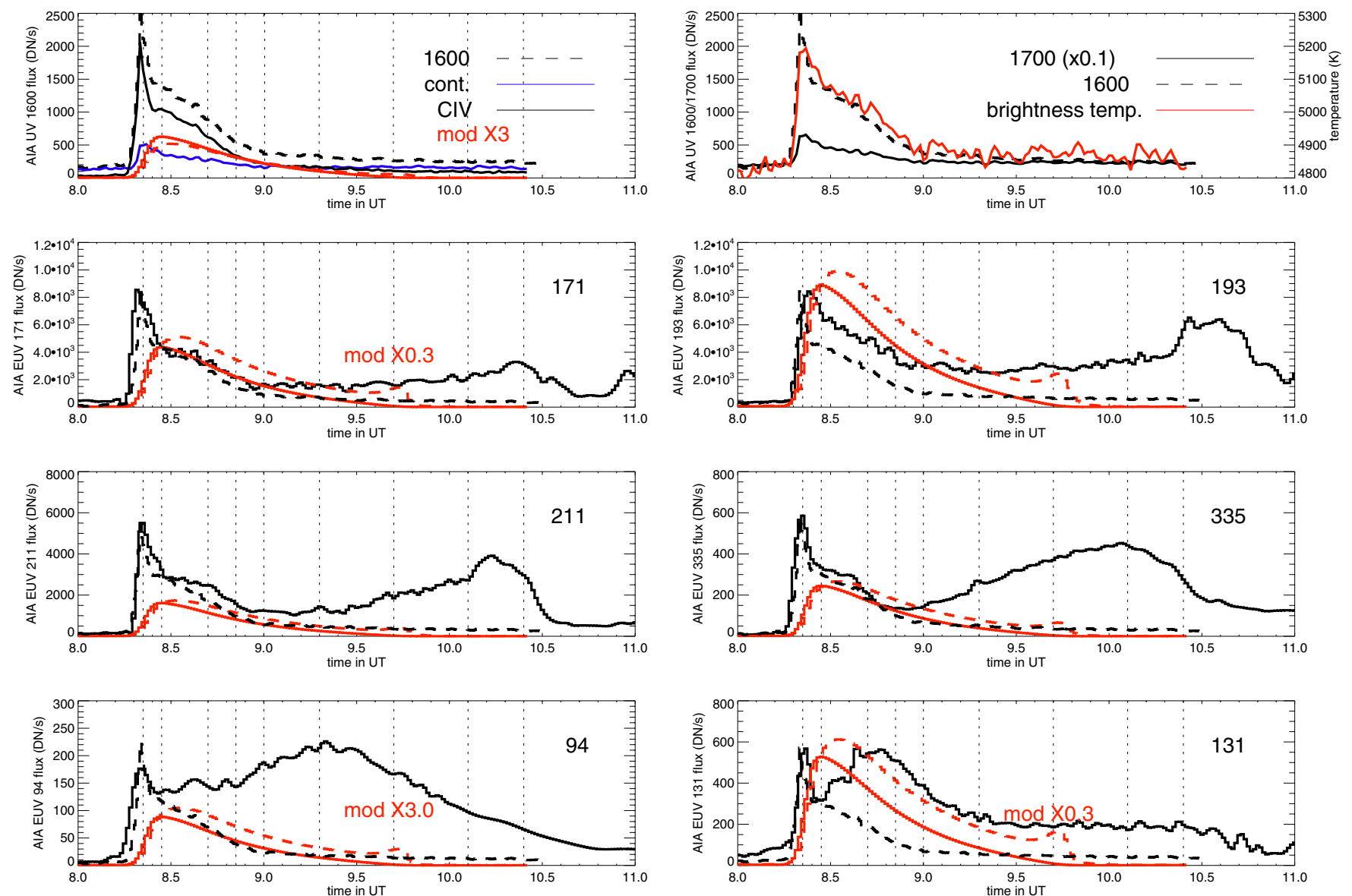

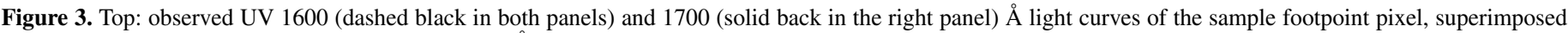

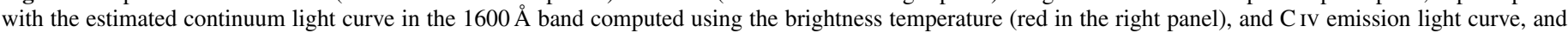

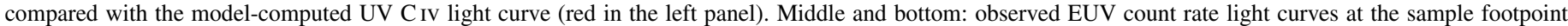

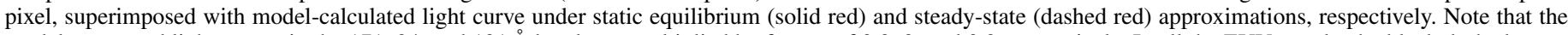

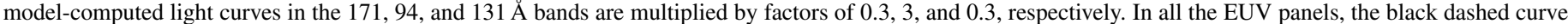
shows the observed UV $1600 \AA$ A light curve arbitrarily scaled. The vertical dotted lines indicate the times of the snapshot images in Figure 2.

(A color version of this figure is available in the online journal.)

more gradual process correlated with plasma evolution in the overlying coronal loop.

The EUV emission, however, exhibits a more complicated structure than the UV light curve at the same footpoint pixel. For example, in the $131 \AA$ band, about 10-20 minutes after the first peak, a second and more gradual emission peak shows up in the EUV light curve. In other EUV bands, the second peak occurs still later by up to $2 \mathrm{hr}$. While the first EUV peak occurs simultaneously in all EUV bands, i.e., independent of temperature, the timing of the second EUV peak is wavelength dependent. In general, emissions at EUV bands sensitive to higher temperatures (e.g., 94, 131, and $335 \AA$ bands with response function peaking at $>3 \mathrm{MK}$ ) tend to rise (when the first peak stops decaying) and peak earlier than the lowtemperature sensitive bands (e.g., 211, 193, and $171 \AA$ bands with response function peaking at $1-2 \mathrm{MK}$ ). These observations convince us that the second-peak EUV emission is explained by the standard picture of post-flare plasma cooling from ten to a few million kelvin.

Moreover, although the second EUV peak is observed in the same pixel as the first peak, in most cases, the two peaks originate from plasmas in different parts of different flare loops. The first peak is from the footpoint of a flare loop formed and heated earlier, and the second peak is a cumulative emission by parts of the loops that are formed progressively and overlap on top of the footpoint of the earlier loop. Figure 2 confirms this scenario by comparing the morphology during the two peaks. It appears that, for the sampled pixel, the first EUV peak occurs at the feet of the set of the short loops residing to the northwest, and the second EUV peak is rather associated with the set of the long loops tending to the south, and these long loops in the south are formed and heated later than the short loops in the north (Qiu et al. 2012). Woods et al. (2011) and Hock et al. (2013) suggest that, in many flares, these long loops associated with what they call the EUV late phase are related to the breakout model for coronal mass ejections.

Figure 4 shows the UV and EUV light curves of the total counts from all footpoint pixels identified in the UV $1600 \AA$ band. It appears that the total EUV light curves also exhibit two or more components. The first component evolves the same way as the UV light curve independent of wavelength or temperature, and the second component evolves on timescales dependent on temperature. Again, the most likely scenario is that the early-phase EUV emissions from these pixels are indeed produced at the flare footpoints in the upper chromosphere or transition region heated impulsively, and emissions later on are from later formed flare loops overlapping the footpoints brightened earlier. The second emission component, even if from a 

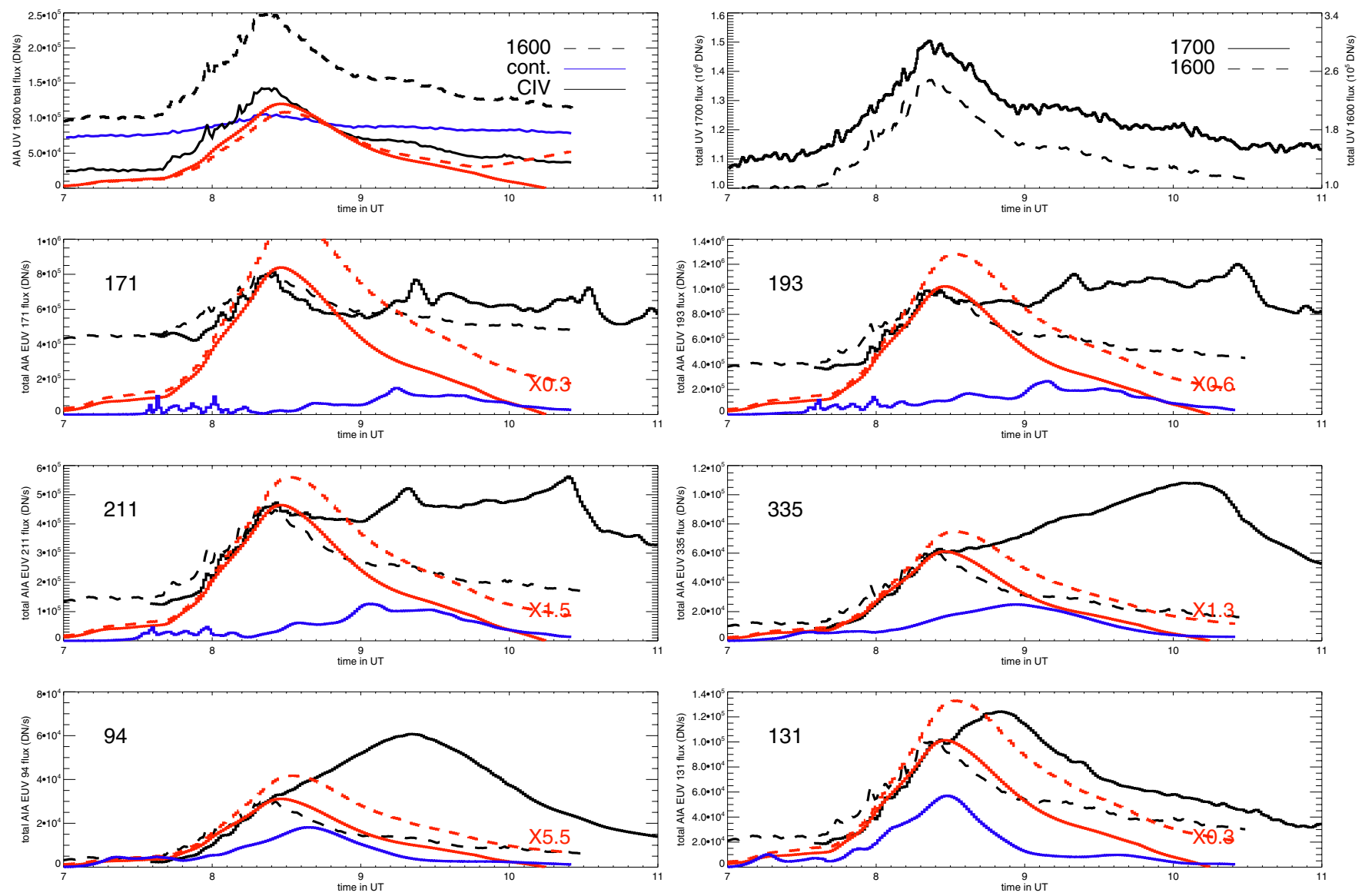

Figure 4. Observed UV and EUV light curves (solid black) summed from all footpoint pixels identified from the UV $1600 \AA$ images, compared with the modelcomputed UV C IV light curve and EUV light curves with static equilibrium (solid red) and steady-state (dashed red) approximations, respectively. Note that the model computed EUV light curves are multiplied by factors indicated in the figure. In all the EUV panels, the black dashed line shows the observed UV $1600 \AA$ A light curve arbitrarily scaled.

(A color version of this figure is available in the online journal.)

single pixel, is a complex collection of coronal emissions from fractions of multiple loops that cannot be easily resolved. In the following text, we focus on discussing the two-phase evolution of the first peak occurred simultaneously in UV and EUV emissions.

\section{FOOTPOINT UV/EUV EMISSION AS A CORONAL PRESSURE GAUGE}

The two phases of the footpoint emission are governed by different physics. The impulsive spike shown in the UV and EUV light curves is considered to be a signature of the lower atmosphere responding to energy deposition. It is most likely generated by a condensation shock front propagating downward from the site of energy deposition by thermal conduction (Fisher 1989). The gradual decay, on the other hand, reflects the cooling of the overlying corona. Hawley et al. (2003) reported such twophase evolution in UV emissions from a few lines in stellar flare observations, and found that these lines (including C IV) can be used as a transition region pressure gauge monitoring evolution of coronal plasmas in overlying flare loops during the cooling phase. During this phase, the entire loop is in approximate hydrostatic balance so that the differential emission measure (DEM) throughout the transition region is proportional to the equilibrium pressure - the coronal pressure. The emission from any line formed at transition region temperatures, such as $\mathrm{C} \mathrm{IV}$, is therefore also proportional to coronal pressure. In the following discussion, we explore whether this pressure-gauge logic can re-produce observed UV and EUV signatures.

\subsection{Transition Region Differential Emission Measure}

To find plasma evolution in overlying coronal loops, Qiu et al. (2012) used a zero-dimensional EBTEL model (Klimchuk et al. 2008; Cargill et al. 2012) to calculate the mean temperature and density in the coronal loop. Inputs to the model include the heating rate and loop length at each footpoint pixel. The latter is measured from the AIA imaging observations. The heating rate is inferred from the impulsive pulse of the UV light curve from that pixel, after using a single scaling parameter. The EBTEL model also allows heat input either directly to the coronal plasma or non-thermal energy deposition in the lower atmosphere. As this particular flare exhibits very little non-thermal signature, we assume that the energy input was of the former variety.

The output of the EBTEL model are coronal plasma properties (mean temperature and density) which are used to compute the synthetic X-ray and EUV emissions by coronal loops observed by GOES and AIA. By matching the observed and synthetic X-ray and EUV fluxes, Qiu et al. (2012) were able to arrive at a first-order estimate to the scaling parameter used to convert impulsive emission to heating. We note that in Qiu et al. (2012), the earlier version of the EBTEL model (EBTEL1; Klimchuk 

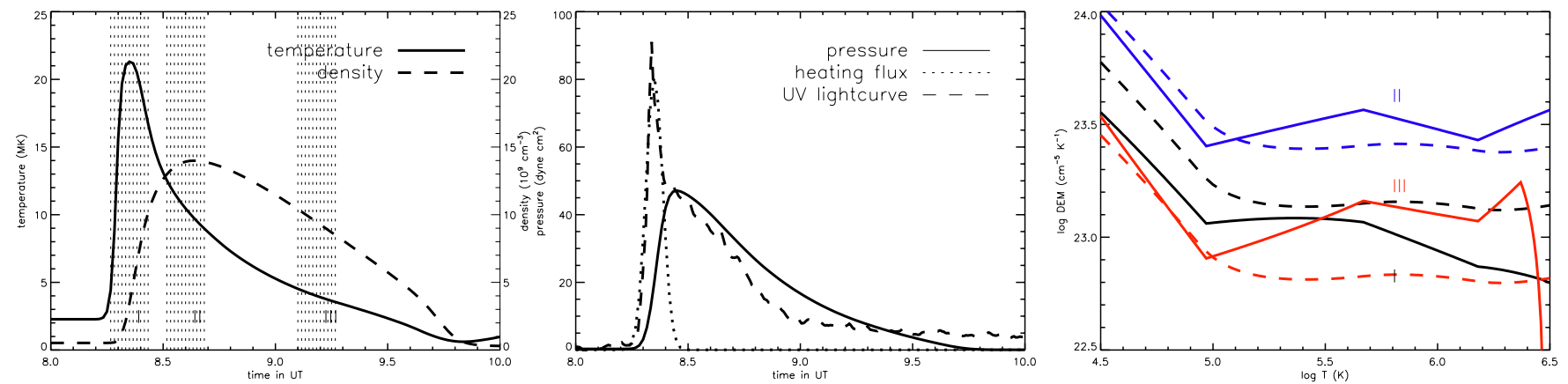

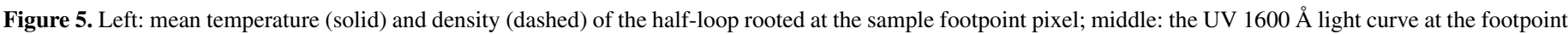

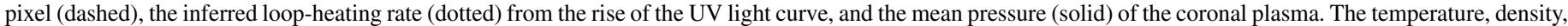

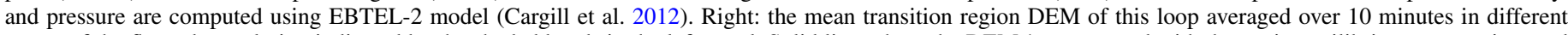

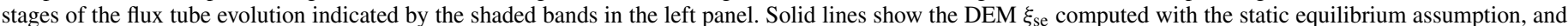
dashed lines show the DEM $\xi_{\text {ss }}$ with the steady-state assumption.

(A color version of this figure is available in the online journal.)

et al. 2008) was employed. In the present study, the coronal plasma properties are recalculated using the updated version of the EBTEL model (EBTEL2; Cargill et al. 2012). The difference between the results by the two versions of the models is insignificant, mainly because of the appropriate choice of free parameters guided by observations.

Figure 5 shows the mean temperature and density of the flux tube rooted at the bright pixel illustrated in Figure 3. The heating rate is inferred from the rise of the UV light curve at this pixel with a duration of 10 minutes, and the best-guess magnitude of the heating flux is $1.6 \times 10^{9} \mathrm{erg} \mathrm{cm} \mathrm{cm}^{-2} \mathrm{~s}^{-1}$. The resultant pressure of the flux tube is plotted in the middle panel, and it is seen that the decay of the UV 1600 light curve (and therefore the EUV light curves as well) evolves on the same timescale as the coronal pressure.

This pressure is used to synthesize the C IV emission following the "pressure gauge" (Fisher 1987; Hawley \& Fisher 1992). We assume the transition region, where the spectral lines form, to be in hydrostatic balance at some pressure. The atmosphere is then structured by the balance between optically thin radiative losses and conductive heat downward from the cooling coronal loop; the plasma flow is neglected. With these conditions, the analytical solution is obtained to compute the DEM along the leg of the flux tube (Fisher 1987; Griffiths et al. 1998; Hawley \& Fisher 1992) to be

$$
\xi_{\mathrm{se}}(T)=\bar{P} \sqrt{\frac{\kappa_{0}}{8 k_{B}^{2}}} T^{\frac{1}{2}} Q^{-\frac{1}{2}}(T),
$$

where

$$
Q(T)=\int_{T_{0}}^{T} T^{\prime \frac{1}{2}} \Lambda\left(T^{\prime}\right) d T^{\prime}
$$

and $\Lambda(T)$ is the optically thin radiative loss function. Expressing the temperature-dependent scaling constant as $g_{\text {se }}(T)$, we can compute the transition region DEM as $\xi_{\text {se }}(T)=g_{\text {se }}(T) \bar{P}$, which is directly proportional to the mean pressure $\bar{P}$ computed using the zero-dimensional EBTEL model (Cargill et al. 2012).

Plasmas inside flaring flux tubes are usually not in static equilibrium but undergo dynamic evolution. During the heating phase, upflow (chromospheric evaporation) up to a few hundred kilometers per second is generated, and the decay phase is dominated by downflow (coronal condensation) of order a few tens of kilometers per second. Therefore, the transition region DEM should be corrected with respect to flow terms; under the steady-state assumption, this is computed as (Klimchuk et al. 2008)

$$
\xi_{\mathrm{ss}}(T)=\bar{P} \frac{\kappa_{0}^{\frac{1}{2}}}{2 k_{B}}\left[T^{\frac{1}{2}} \Lambda(T)\right]^{-\frac{1}{2}}\left(\sqrt{\gamma^{2}+1}+\gamma\right),
$$

where $\gamma$ is a function of mean coronal temperature $\bar{T}_{c}$ and flow speed $v_{c}$ across the coronal base, both calculated in EBTEL:

$$
\gamma=\frac{5 k_{B} T^{\frac{1}{2}}}{2 \sqrt{\kappa_{0} T^{\frac{1}{2}} \Lambda(T)}} \frac{-v_{c}}{\bar{T}_{c}} .
$$

The above pressure-gauge relation may be written as $\xi_{\mathrm{ss}}(T)=$ $g_{\text {ss }}(T) \bar{P}$. Similar to Equation (1), the transition region DEM is scaled with the coronal pressure, the scaler $g_{\text {ss }}$ being dependent also on the plasma flow. For upflow, $v_{c}>0$, and for downflow, $v_{c}<0$.

The right panel in Figure 5 shows the transition region DEM $\xi_{\text {se }}$ and $\xi_{\text {ss }}$ in a few stages during the flux tube evolution. These few stages are indicated by the shaded bands in the left panel, representative of the rise, early-decay, and late-decay phases of the flux tube, respectively. The DEM in each stage is the mean value over 10 minutes. The DEM is modified when flow is included.

During the impulsive heating phase, the loop is far from equilibrium and cannot be modeled in this manner. Rapid heating of the lower atmosphere from thermal conduction leads to upward and downward moving shocks (MacNeice 1986). The upward shock is the leading edge of an evaporation flow of a hundred $\mathrm{km} \mathrm{s}^{-1}$ or more. The downward shock leads downflow, often called chromospheric condensation (Fisher 1989) in this initial phase of the flare, which is distinguished from the process of cooling and draining of coronal plasma in the later phase. The effect, sometimes regarded as a rapid downward motion of the transition region, is to raise material at chromospheric densities to transition region temperatures. This results in emission from associated lines, such as C IV, far in excess of what an equilibrium atmosphere might produce. This enhancement lasts only as long as the condensation shock does. Afterward the loop assumes hydrostatic balance at its new pressure and begins cooling as described above. We attribute the brief, impulsive enhancement in C IV to this scenario and use its amplitude and duration to infer the energy input into the 

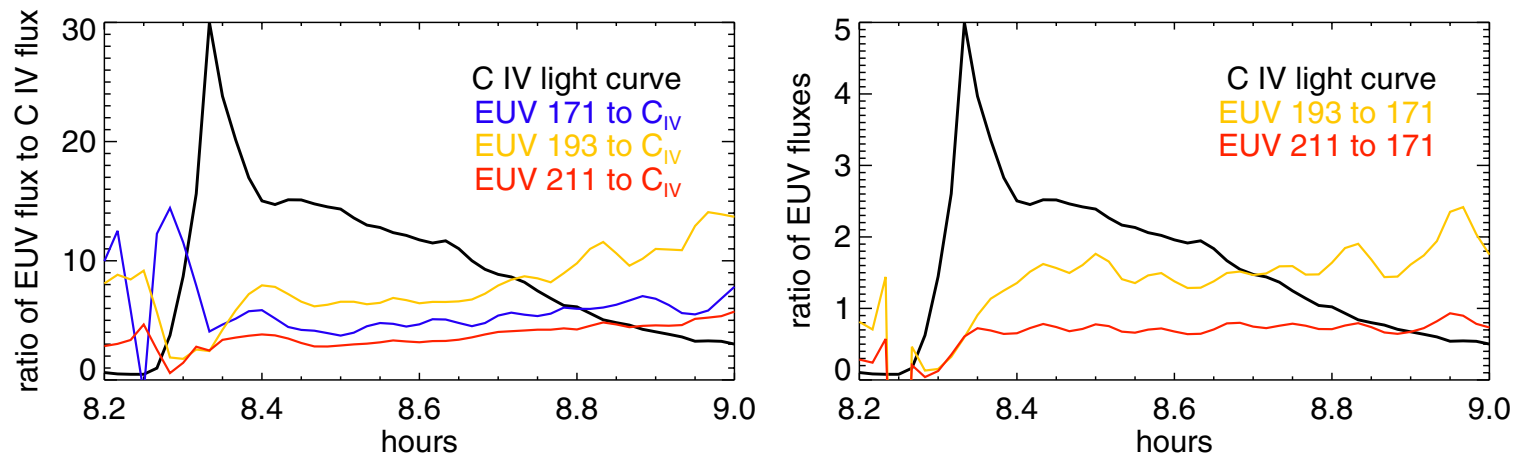

Figure 6. Left: ratio of observed EUV fluxes in 171, 193, and $211 \AA$ A bands to the C IV flux for the sample pixel during the flare. Right: ratio of observed EUV fluxes in 193 and $211 \AA$ A bands to that in $171 \AA$ A band for the same pixel. In both panels, the C IV light curve, arbitrarily scaled, is plotted to provide information of the evolution of the footpoint emission.

(A color version of this figure is available in the online journal.)

coronal loop, but do not attempt to capture the physics in the EBTEL model. Instead we quantify the relationship through a single empirical parameter, which we fix through observational comparison as described above.

\subsection{Comparison with Observations}

To compare with observations, we use the calculated transition region DEM to compute C IV at the flaring pixel. The emissivity of the optically thin C IV line $\epsilon(T)$ is derived from CHIANTI 7.0 with ionization equilibrium (Dere et al. 1997; Landi et al. 2012). The total C IV photon flux in units of photons $\mathrm{cm}^{-2} \mathrm{~s}^{-1} \mathrm{sr}^{-1}$ is computed using the DEM, and is converted to observed count rate in units of $\mathrm{DN} \mathrm{s}^{-1}$ by convolving with the AIA instrument response function. Note that we have used the latest version of the response function released in 2012 January, and with the correction factor from normalization to EVE observations; for the $1600 \AA$ band, this correction factor is 2.1 , and for the $1700 \AA$ band, this correction factor is 0.75 (Boerner et al. 2012).

This is then compared with the observed C IV light curve. Comparison for a single pixel is given in the top left panel in Figure 3, and the summed emission from all footpoint pixels is shown in the top left panel in Figure 4. It is seen that, during the impulsive rise, the model-calculated C IV emission is far less than observed. This is expected from the shock condensation scenario outlined in the previous section. On the other hand, during the decay, the model-calculated emission declines on the same timescale as observed; the amount of emission, computed with either static equilibrium or steady-state approximation, is smaller than the observed flux by a factor of two to three for the bright pixel. When emissions from all pixels are summed up, the computed C IV emission agrees with the observed total. This result indicates that the pressure-gauge approximation can reproduce the observed decay timescale reasonably well; on the other hand, the magnitude comparison for single pixel and for all the pixels suggest that the observation or model or both of the C IV emission seem to differ for differently heated flux tubes.

In the same way, we convolve the AIA instrument response functions of the six EUV bands with the computed DEM to synthesize the EUV count rate light curves at the flare footpoints - the $304 \AA$ band is not computed since the formation mechanism of this line is more complex, for example, it is not optically thin. Figure 3 shows comparison of the EUV light curves for one pixel, and Figure 4 shows the sum of the emissions in all footpoint pixels. In these figures, the solid red curves and dashed red curves show the computed flux with static or steady-state approximations, respectively. In the later case, the computed flux is enhanced as coronal downflow into the transition region is included in the decay phase.

In synthesizing the EUV bands we integrate the DEM from 100,000 to $500,000 \mathrm{~K}$ only; we do not include the corona. The upper bound of the temperature is rather arbitrary but not entirely unreasonable. The temperature distribution of the plasmas is along the length of the flare loop; however, we only look at one pixel at the footpoint. Because of the geometry of the loop on the solar disk, and the fact that flare loops in this event are very long with their half-length ranging from 50-100 Mm, only relatively cool plasmas at the bottom of the flux tube would contribute to emission at the footpoint pixel.

The figures show that, again, the computed and observed EUV light curves for the first peak decay on almost the same timescale, which is the decay timescale of the pressure in the flux tube. In terms of magnitude, the computed emission is quite comparable with the observed in 335, 211, and $193 \AA$ A bands. The computed flux in 131 and $171 \AA$ bands is higher by a factor of three to four; on the other hand, the computed flux in $94 \AA$ band is smaller than observed by nearly a factor of five. Note that Brosius \& Holman (2012) also conjectured that the lowtemperature response in the $94 \AA$ band is likely underestimated by a factor of five. With uncertainties in the effective upperbound temperature that contributes to the footpoint emission, as well as in the low-temperature response of AIA filters, it is still striking that the pressure-gauge calculation based on very simplified assumptions produces close estimates of the UV and EUV emissions at the flare footpoints.

The above experiments show that the pressure-gauge calculation may be applied to the gradual cooling phase when the flare loop is very close to equilibrium. On the other hand, the calculation does not agree with the signatures during the impulsive heating phase, which is unlikely to be in an equilibrium state. The static or steady-state equilibrium dictates that the plasma DEM is proportional to the pressure which is uniform along the loop. Therefore, the ratio of optically thin EUV or UV fluxes should remain a constant during its evolution. In Figure 6, we plot the ratio of the EUV flux in a few bands to the C IV flux as well as the ratio of EUV fluxes for the sample footpoint pixel during its evolution. It is shown that the flux ratio is almost a constant during the gradual cooling phase, justifying the pressure-gauge assumptions. However, during the impulsive heating phase, the flux ratio varies rapidly. Such behavior is observed in most footpoint pixels. It is therefore evident 
that the impulsive phase cannot be described by steady-state equilibrium.

Finally, to estimate the contribution of coronal emission from flare loops on top of the footpoint pixels, we plot the synthetic EUV emission by plasmas in flare loops (Qiu et al. 2012) but along a length of only 1 pixel. These are shown in the blue curves in the middle and bottom panels in Figure 4 . It is evident that in nearly every band the coronal emission is delayed with respect to the footpoint emission. The peak of the coronal emission component is also temperature dependent, with hightemperature emission (131 and $94 \AA$ bands) peaking earlier than low-temperature emissions (211, 193, and $171 \AA$ A bands). During the first peak of the observed emission, the contribution by the coronal component is insignificant except in the relatively hot bands, for example, in the $131 \AA$ band.

\section{CONCLUSIONS AND DISCUSSIONS}

We have investigated the UV and EUV footpoint emissions observed by AIA during the early phase of the flare. It is recognized that UV emission of the flare occurs at footpoints. We have shown that these same footpoints also produce EUV emissions observed by AIA, whose evolution is nearly identical to the UV light curve with a rapid rise on timescales of a few minutes followed by a gradual decay over a few tens of minutes in this long-duration flare. Therefore, these emissions are most likely produced by the same mechanism: impulsive heating of the lower atmosphere-the upper chromosphere and transition region-from a downward thermal conduction flux, and subsequent decay governed by the coronal plasma hydrodynamic evolution.

Using a simple zero-dimensional loop heating model and loop heating rates empirically inferred from the rapid UV pulse, we calculate mean properties of plasmas inside flaring loops, and in turn, compute the transition region DEM as scaled to the coronal pressure with static or steady-state approximations. It is shown that the computed footpoint emissions in UV and EUV bands exhibit the same evolutionary timescale as observed, which is the timescale of the coronal pressure. Assuming that the observed photon flux is produced by plasmas at the coronal base with relatively low temperatures up to a few hundred thousand kelvin, the amount of computed emission compares well with the observed in the seven bands by a factor of three to five at most, a fairly good agreement given uncertainties in the loop geometry and the AIA response functions at low temperatures. This simple exercise suggests that evolution of flare footpoint emissions may be used to monitor coronal plasma evolution and shows the importance of coupling the coronal and lower atmosphere heating and dynamics as independent constraints to loop heating models.

It is noted that the transition region DEM may be substantially increased at temperatures beyond a few hundred thousand kelvin. Computed EUV flux taking into account these higher temperature plasmas, however, produces a lot more flux than observed by one to two orders of magnitude. This may indicate either a temperature-dependent filling factor of this order or that the static or steady-state assumptions are not a good approximation for plasmas at higher temperatures.

The above experiment does not reproduce the impulsive pulse of UV and EUV emissions in the first few minutes, indicating that steady-state assumptions and/or assumed equilibrium conditions used to calculate UV and EUV lines in CHIANTI are not adequate for this period of impulsive heating. It is also plausible that non-thermal particles heat the lower atmosphere during this phase; however, there is no strong evidence for the presence of these particles in this event. A more sophisticated hydrodynamic modeling aided with imaging spectroscopic observations of the flare footpoints will help gain insight into this phase.

We thank Dr. R. C. Canfield for illuminating us about the flare energetics and lower-atmosphere heating. We thank the referee for a careful reading of the manuscript and constructive comments that help improve clarity of the paper. We acknowledge $S D O$ for providing quality observations. The work by J.Q. and W.L. is supported by NSF grant ATM-0748428. Part of the work was conducted during the NSF REU Program at Montana State University. D.W.L. acknowledges support by the NASA Supporting Research and Technology Program. The work of J.A.K. was supported by the NASA Supporting Research and Technology Program.

\section{REFERENCES}

Antonucci, E., Gabriel, A. H., Acton, L. W., et al. 1982, SoPh, 78, 107 Bentley, R. D., Doschek, G. A., Simnett, G. M., et al. 1994, ApJL, 421, L55 Boerner, P., Edwards, C., Lemen, J., et al. 2012, SoPh, 275, 41

Brekke, P., \& Kjeldseth-Moe, O. 1994, SoPh, 150, 19

Brekke, P., Rottman, G. J., Fontenla, J., \& Judge, P. G. 1996, ApJ, 468, 418 Brosius, J. W., \& Holman, G. D. 2012, A\&A, 540, A24

Canfield, R. C. 1986, in Proc. Solar Maximum Mission Symposium, The Lower Atmosphere of Solar Flares, ed. D. F. Neidig (Sunspot, NM: National Solar Observatory), 10

Canfield, R. C., \& Gayley, K. G. 1987, ApJ, 322, 999

Canfield, R. C., \& Metcalf, T. R. 1987, ApJ, 321, 586

Canfield, R. C., Metcalf, T. R., Strong, K. T., \& Zarro, D. M. 1987, Natur, 326,165

Canfield, R. C., Metcalf, T. R., Zarro, D. M., \& Lemen, J. R. 1990a, ApJ, 348,333

Canfield, R. C., Penn, M. J., Wulser, J.-P., \& Kiplinger, A. L. 1990b, ApJ, 363,318

Cargill, P. J., Bradshaw, S. J., \& Klimchuk, J. A. 2012, ApJ, 752, 161

Cheng, C.-C., \& Pallavicini, R. 1987, ApJ, 318, 459

Cheng, J. X., Kerr, G., \& Qiu, J. 2012, ApJ, 744, 48

Cook, J. W., \& Brueckner, G. E. 1979, ApJ, 227, 645

Culhane, J. L., Fludra, A., Bentley, R. D., et al. 1992, PASJ, 44, L101

Culhane, J. L., Harra, L. K., James, A. M., et al. 2007, SoPh, 243, 19

Del Zanna, G., Mitra-Kraev, U., Bradshaw, S. J., Mason, H. E., \& Asai, A. 2011, A\&A, 526, A1

Dere, K. P., Landi, E., Mason, H. E., Monsignori Fossi, B. C., \& Young, P. R. 1997, A\&AS, 125, 149

Ding, M. D., Fang, C., \& Huang, Y. R. 1995, SoPh, 158, 81

Doschek, G. A., Mariska, J. T., Watanabe, T., et al. 1992, PASJ, 44, L95

Doyle, J. G., \& Phillips, K. J. H. 1992, A\&A, 257, 773

Fisher, G. H. 1987, ApJ, 317, 502

Fisher, G. H. 1989, ApJ, 346, 1019

Fisher, G. H., Canfield, R. C., \& McClymont, A. N. 1985, ApJ, 289, 414

Fisher, G. H., \& Hawley, S. L. 1990, ApJ, 357, 243

Graham, D. R., Fletcher, L., \& Hannah, I. G. 2011, A\&A, 532, A27

Graham, D. R., Hannah, I. G., Fletcher, L., \& Milligan, R. O. 2013, ApJ, 767,83

Griffiths, N. W., Fisher, G. H., \& Siegmund, O. H. W. 1998, in ASP Conf. Ser 154, Cool Stars, Stellar Systems, and the Sun, ed. R. A. Donahue \& J. A Bookbinder (San Francisco, CA: ASP), 621

Handy, B. N., Acton, L. W., Kankelborg, C. C., et al. 1999, SoPh, 187, 229

Harra, L. K., Hara, H., Imada, S., et al. 2007, PASJ, 59, 801

Harrison, R. A., Sawyer, E. C., Carter, M. K., et al. 1995, SoPh, 162, 233

Hawley, S. L., Allred, J. C., Johns-Krull, C. M., et al. 2003, ApJ, 597, 535

Hawley, S. L., \& Fisher, G. H. 1992, ApJS, 78, 565

Hock, R. A., Woods, T. N., Klimchuk, J. A., Eparvier, F. G., \& Jones, A. R. 2013, ApJ, in press (arXiv:1202.4819)

Ichimoto, K., \& Kurokawa, H. 1984, SoPh, 93, 105

Klimchuk, J. A. 2009, in ASP Conf. Ser. 415, The Second Hinode Science Meeting: Beyond Discovery-Toward Understanding, ed. B. Lites, M. Cheung, T. Magara, J. Mariska, \& K. Reeves (San Francisco, CA: ASP), 221

Klimchuk, J. A. 2012, JGRA, 117, 12102

Klimchuk, J. A., Patsourakos, S., \& Cargill, P. J. 2008, ApJ, 682, 1351 
Landi, E., Del Zanna, G., Young, P. R., Dere, K. P., \& Mason, H. E. 2012, ApJ, 744, 99

Lemen, J. R., Title, A. M., Akin, D. J., et al. 2012, SoPh, 275, 17

Liu, W.-J., Qiu, J., Longcope, D. W., \& Caspi, A. 2013, ApJ, 770, 111

Machado, M. E., \& Henoux, J.-C. 1982, A\&A, 108, 61

MacNeice, P. 1986, SoPh, 103, 47

Milligan, R. O. 2011, ApJ, 740, 70

Milligan, R. O., \& Dennis, B. R. 2009, ApJ, 699, 968

Milligan, R. O., Gallagher, P. T., Mathioudakis, M., \& Keenan, F. P. 2006a, ApJL, 642, L169

Milligan, R. O., Gallagher, P. T., Mathioudakis, M., et al. 2006b, ApJL, 638, L117

Mrozek, T., \& Tomczak, M. 2004, A\&A, 415, 377

Patsourakos, S., \& Klimchuk, J. A. 2008, ApJ, 689, 1406

Phillips, K. J. H., Bromage, G. E., \& Doyle, J. G. 1992, ApJ, 385, 731
Qiu, J., Hu, Q., Howard, T. A., \& Yurchyshyn, V. B. 2007, ApJ, 659, 758

Qiu, J., Liu, W., Hill, N., \& Kazachenko, M. 2010, ApJ, 725, 319

Qiu, J., Liu, W.-J., \& Longcope, D. W. 2012, ApJ, 752, 124

Raftery, C. L., Gallagher, P. T., Milligan, R. O., \& Klimchuk, J. A. 2009, A\&A, 494, 1127

Reale, F., Landi, E., \& Orlando, S. 2012, ApJ, 746, 18

Schmieder, B., Forbes, T. G., Malherbe, J. M., \& Machado, M. E. 1987, ApJ, 317,956

Tsuneta, S., Acton, L., Bruner, M., et al. 1991, SoPh, 136, 37

Watanabe, T., Hara, H., Sterling, A. C., \& Harra, L. K. 2010, ApJ, 719, 213

Woods, T. N., Hock, R., Eparvier, F., et al. 2011, ApJ, 739, 59

Wuelser, J.-P., Canfield, R. C., Acton, L. W., et al. 1994, ApJ, 424, 459

Wuelser, J.-P., \& Marti, H. 1989, ApJ, 341, 1088

Zarro, D. M., Canfield, R. C., Metcalf, T. R., \& Strong, K. T. 1988, ApJ, 324, 582 